\title{
Periodontal Health, Quality of Life, and Smiling Patterns - An Exploration
}

Ruchir R. Patel, * Philip S. Richards, ${ }^{\dagger}$ and Marita R. Inglehart ${ }^{\dagger}$

Background: This study explored whether periodontal health/ disease affects psychosocial outcomes in smiling patterns of particular subjects and their smile-related quality of life.

Methods: We collected data from 21 regularly scheduled patients in a periodontal graduate student clinic (four males and 17 females; average age: 50.38 years; age range: 24 to 82 years). The subjects were videotaped while watching a funny television (TV) program. Two independent raters rated each videotape at 31 predetermined time points to assess four aspects of the objective smiling patterns of the subjects. In addition, the subjects responded to a questionnaire to assess their smile-related quality of life. Provider ratings and chart review data were used to assess the clinically assessed oral health status of the subjects.

Results: The smile-related quality of life of the subjects correlated significantly with indicators of the periodontal health of the subjects, such as the number of mobile teeth $(r=0.681 ; P=$ $0.000)$, missing teeth $(r=0.784 ; P=0.001)$, and gingival recession in the esthetic zone $(r=0.718 ; P=0.001)$. Periodontal health and smiling patterns also were correlated. The more teeth with probing depths between 4 and $6 \mathrm{~mm}$ the subjects had, the less widely they opened their mouths when they smiled ( $r=-0.468 ; P=0.032)$; the more hypermobile teeth the subjects had, the less open their smiles were $(r=-0.442$; $P=0.045)$ and the more likely they were to cover their mouths when they smiled $(r=0.517 ; P=0.017)$; and the more sites of gingival recession in the esthetic zone the subjects had, the fewer teeth they showed when they smiled $(r=-0.491 ; P=$ 0.028).

Conclusions: The periodontal health of the subjects affects their smiling patterns and their smile-related quality of life. Poor periodontal health may prevent adults from expressing positive emotions which, in turn, can impact their self-concept as well as their social interactions. J Periodontol 2008;79:224231.

\section{KEY WORDS}

Oral health; periodontal disease; periodontitis; quality of life; smiling.

* School of Dentistry, University of Michigan, Ann Arbor, MI.

$\dagger$ Department of Periodontics and Oral Medicine, School of Dentistry, University of Michigan.
I n 2000, the United States Surgeon General published the first ever Surgeon General's report on oral health. ${ }^{1}$ This report documented the prevalence of periodontal disease in $>50 \%$ of the adult United States population and stressed that periodontal disease was especially widespread among socioeconomically disadvantaged citizens. One of the major recommendations of this important document was to challenge oral health care providers to consider the many ways in which oral health affects patients' quality of life. The present study followed this recommendation and focused on one particular aspect of the important relationship between oral health and quality of life by exploring how periodontal disease affects the smiling patterns of the subjects and their self-reported smile-related quality of life.

Oral health-related quality of life (OHRQoL) is a relatively new concept in the oral health sciences literature. ${ }^{2}$ It is defined as that part of a person's quality of life that is affected by one's oral health status. Specifically, it includes how oral health affects a person's functioning, such as chewing, biting, and speaking; experiences of pain/discomfort; and psychologic and social well-being. ${ }^{3}$ Many studies have been conducted concerning the relationships between OHRQoL and dentures, ${ }^{4-6}$ orthodontics, ${ }^{7,8}$ and caries. ${ }^{9-11}$ Studies ${ }^{12-14}$ also have explored how periodontal disease affects OHRQoL. In 2004, Needleman et al. ${ }^{12}$ analyzed OHRQoL data and data from a comprehensive periodontal examination of $>200$ 
adult subjects and found that the effect of oral health on quality of life was considerable. The OHRQoL scores of the subjects correlated significantly with the number of teeth with probing depths $\geq 5 \mathrm{~mm}$. Therefore, the investigators concluded that periodontal status impacted the quality of life of the subjects. In 2006, $\mathrm{Ng}$ and Leung ${ }^{13}$ provided additional evidence that periodontal health/disease and OHRQoL are related. These investigators analyzed data from 767 adults who had been assigned to a "healthy/low periodontal attachment level subject group" (full-mouth mean clinical attachment level [CAL] $\leq 2 \mathrm{~mm}$ ) or to a "high/severe periodontal attachment loss group" (full-mouth mean CAL $>3 \mathrm{~mm}$ ). A comparison of the average OHRQoL scores of these two groups of subjects showed significant differences concerning the degrees to which they reported functional limitations, physical pain, psychologic discomfort, and physical and psychologic disabilities. Therefore, the investigators concluded that their results showed that there is a significant association between OHRQoL and periodontal disease. Most recently, in April 2007, Cunha-Cruz et al. ${ }^{14}$ explored the relationship between subjects' self-reports of their OHRQoL and clinical indicators of periodontal disease. These investigators found that OHRQoL and self-perceptions of oral health status were related significantly to the periodontal status of the subjects as measured by having more than eight teeth with probing depth $>5 \mathrm{~mm}$. Therefore, they concluded that oral health-related problems of patients who visit a periodontist negatively affect their quality of life.

The psychologic and social components of the OHRQoL of subjects are mostly related to the appearance of their teeth and especially the way they evaluate their smiles. It might seem that the attractiveness of a person's smile primarily is affected by malocclusion. However, Patel et al. ${ }^{15}$ showed in a study with 4 - to 12 -year-old children that their objectively assessed smiling patterns were significantly related to several other indicators of their clinically assessed oral health. For example, children without caries evaluated their own smiles more positively, showed more teeth when smiling, and received more positive parent evaluations for their smiles than children with decay. Therefore, these investigators concluded that poor oral health is related significantly to children's smiling patterns and the way others perceived their smiles. Although this study ${ }^{15}$ may have shown significant relationships between caries and smiling patterns in children, it did not address the relationships between smile-related quality of life and periodontal health indicators. Therefore, the present study addressed whether adults' periodontal health status is related to their smile-related quality of life and their smiling patterns.

Why is it important to understand whether there is a relationship between periodontal health and these psychosocial outcomes? Smiling affects how others perceive a person and, thus, this person's social interactions, ${ }^{16}$ mood, and self-confidence. ${ }^{16,17}$ For example, in 1990, Reis et al. ${ }^{16}$ published an article with the provocative title "What is Smiling is Beautiful and Good." This study showed the significant role that smiling plays for others' evaluations of a person and specifically that smiling affected judgments of physical attractiveness and other characteristics typically ascribed to attractive persons. In particular, smiling faces were evaluated as being more sincere, more sociable, and more competent than non-smiling faces. Overall, these findings clearly demonstrated the ways in which positive smiling patterns affect how others evaluate a person. In addition to documenting the effects of smiling on social interactions, research also documented how subjective well-being and selfesteem can be affected by a person's smiling pattern. For example, Källestål et al. ${ }^{18}$ showed that a confident smile was linked to a person's positive self-esteem, self-confidence, and overall well-being. More specifically, Low et al. ${ }^{9}$ showed that children with worries about their teeth and children with missing, stained, or decayed teeth were less confident about smiling.

Given the important effects that smiling has on a person's life, it would be interesting to understand whether there is a relationship between periodontal health/disease and the smile-related quality of life of subjects and their objectively assessed smiling patterns. Figure 1 depicts the topics addressed in this study.

\section{MATERIALS AND METHODS}

This study was approved by the Institutional Review Board for the Health Sciences at the University of Michigan. The research was conducted at the Graduate Periodontal Clinic, School of Dentistry, University of Michigan, between June 1, 2006 and August 15, 2006.

\section{Respondents}

Twenty-one patients from the graduate periodontal clinic participated in this study. All participants were

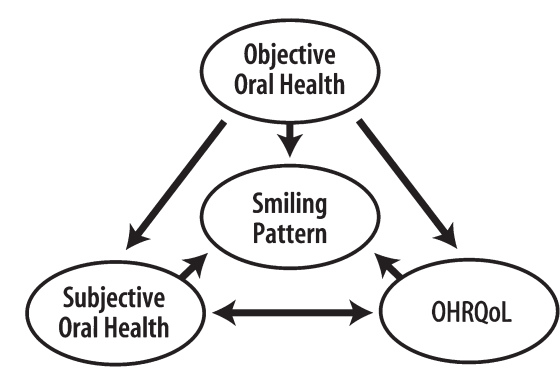

Figure I.

Overview of the explored relationships. 
healthy, and there were no language barriers. The four male and 17 female subjects ranged in age from 24 to 82 years (mean \pm SD: $50.38 \pm 18.717$ ). To be eligible to participate in the study, the subjects had to be regularly scheduled periodontal subjects $>18$ years, and they had to be able to watch a video without being distracted.

\section{Procedure}

Regularly scheduled patients were invited to participate in this research upon arrival at the graduate periodontal clinic. They were informed that they would receive free parking as an incentive for their participation. Following written consent to participate in the study and after signing the Health Insurance Portability and Accountability Act form, the subjects responded to a survey concerning their oral health and their oral health-related and smile-related quality of life. The subjects then watched a short (6 minutes long) tape of an episode of the "Seinfeld" television series in a room by themselves. While they watched the video, their faces were videotaped with a digital camera that was mounted on top of the television (TV) set. The digital recordings of the subjects' faces were converted into digital video discs (DVDs) to make it possible to watch them on computer screens. Two trained independent raters evaluated these videos. The subjects' periodontal clinicians assessed the current periodontal health of the subjects as well as the expected outcome of the periodontal treatment (= subjective periodontal health assessment). In addition, the dental charts of the subjects were reviewed to assess their periodontal health.

\section{Materials}

Four sets of data were collected: subjects' OHRQoL indicators, subjective provider assessments of the periodontal health status and expected treatment outcomes of the subjects, clinically assessed periodontal health indicators, and the smiling patterns of the subjects.

The OHRQoL and smile-related quality of life of the subjects were assessed with six items that had a Likert scale answer format. The three statements "My teeth and gums cause discomfort," "My teeth and gums reduce my general happiness with life," and "My teeth and gums affect my life in all of its aspects" were used as general OHRQoL indicators. The three items "My teeth and gums keep me from smiling," "I like my smile," and "I am happy with the looks of my teeth and gums" were used as indicators of the smilerelated quality of life of the subjects. The respondents indicated on a five-point scale ranging from $1=$ "disagree strongly" to 5 = "agree strongly" how much they disagreed/agreed with each statement. These items originally were used as part of the Michigan Oral Health-Related Quality of Life Scale-Adult Version. ${ }^{19}$
The subjective provider assessments of the periodontal health status and expected treatment outcomes of the subjects were measured with two items: "Considering all aspects of periodontal status/ health, how would you describe the patient's current status?" and "How would you describe the likely health outcomes (overall prognosis) of the periodontal treatment planned for this patient?" The answers to these two items were given on a four-point answer scale: 1 = excellent; 2 =good; $3=$ fair; and $4=$ poor.

Data concerning six clinically assessed indicators of the periodontal health of the subjects were collected in a chart review. These periodontal indicators were the number of teeth 1 ) with 4 to $6 \mathrm{~mm}$ probing depth, 2 ) with $\geq 6 \mathrm{~mm}$ probing depth, 3) with mobility, 4) that were missing (no third molars), 5) with recession in the esthetic zone, and 6) with restorations.

Finally, the smiling patterns of the subjects were assessed while the subjects watched 6 minutes of an episode of "Seinfeld," a popular TV comedy. A segment of these subject recordings, which started 5 seconds before a particularly funny sequence of the TV episode began and lasted for 2 minutes and 30 seconds, was transferred to a DVD. Two raters watched these DVDs independently and measured each subject's smile before the funny sequence and at 30 specific time points after it began. The first measurement was a baseline measurement at the beginning of the DVD tape just before a sound occurred that indicated that the funny segment of the video started. The next 30 measurements were spaced every 5 seconds from the time the funny sequence started. For each measurement point, the raters measured three indicators: the width of the subject's mouth in millimeters, the opening of the subject's mouth in millimeters, and the number of teeth shown. These three indicators were chosen based on considerations concerning the measurement of facial expressions. ${ }^{20}$ In addition, the raters also noted whether the subject covered his/her mouth with a hand while watching the TV program. The measurements of the subject's mouth width were standardized by dividing each of a subject's 30 measurements by the subject's baseline measurement. Thus, a score $>1$ represented a wider mouth than measured at baseline. Then, an average standardized "width" score for the 30 measurements of each subject was determined for each rater. The interrater reliabilities of the "width" scores were $r=0.92$. The "width" rating scores of the two raters were averaged and used as an indicator of the subject's "width of mouth." In addition, an average "mouth opening" score, a sum score of the "number of teeth shown," and a sum score of "covering the mouth" were determined for each subject based on the 30 ratings for each rater separately. The interrater reliabilities of these scores were high $(r=0.88, r=0.99$, and $r=0.96$, respectively). Thus, 
the scores of the two raters were averaged, and the mean ratings were used as indicators of the videotaped smile ratings.

\section{Statistical Methods}

Descriptive statistics are provided in Table 1 to allow the reader to gain a sense of how the different characteristics were distributed in this sample. In addition, correlations between the different indicators were computed to explore the relationships present in the data.

\section{RESULTS}

Table 1 provides an overview of the frequency distributions of the various characteristics studied plus measures of central tendency and variability. Concerning the periodontal/oral health indicators, it is evident that the range of experiences is large, given that for each indicator, there were subjects without any signs of periodontal disease on one end of the spectrum, and there were subjects with a considerable number of teeth with poor oral health on the other end. Not surprisingly, the providers' subjective

Table I.

Overview of the Periodontal Health of Subjects, Provider Assessments, and Smile-Related Quality-of-Life Response of Subjects

\begin{tabular}{|c|c|c|c|c|c|c|}
\hline & \multicolumn{4}{|c|}{ Number of Teeth } & \multirow{2}{*}{$\begin{array}{l}\text { Average Number } \\
\text { of Teeth }\end{array}$} & \multirow[b]{2}{*}{ SD (range) } \\
\hline & 0 & I to 2 & 3 to 6 & $7+$ & & \\
\hline \multicolumn{7}{|l|}{ Periodontal health indicator } \\
\hline Probing depth 4 to $6 \mathrm{~mm}$ & $14 \%$ & $24 \%$ & $57 \%$ & $5 \%$ & 3.38 & 2.48 (0 to 10$)$ \\
\hline Probing depth $\geq 6 \mathrm{~mm}$ & $24 \%$ & $19 \%$ & $33 \%$ & $24 \%$ & 3.57 & 3.06 (0 to 8$)$ \\
\hline Mobility & $62 \%$ & $10 \%$ & $19 \%$ & $10 \%$ & 1.62 & $2.50(0$ to 7$)$ \\
\hline Missing teeth (no third molars) & $14 \%$ & $33 \%$ & $43 \%$ & $10 \%$ & 3.62 & $3.38(0$ to 14$)$ \\
\hline Gingival recession in esthetic zone & $52 \%$ & $29 \%$ & $19 \%$ & $0 \%$ & 1.33 & $1.74(0$ to 6$)$ \\
\hline \multirow[t]{2}{*}{ Restorations } & $10 \%$ & $24 \%$ & $19 \%$ & $48 \%$ & 6.29 & 4.46 (0 to I5) \\
\hline & $4=$ Poor & $3=$ Fair & $2=$ Good & $\mathrm{I}=$ Excellent & Mean & SD (range) \\
\hline \multicolumn{7}{|l|}{ Provider assessments } \\
\hline Current periodontal health status & $10 \%$ & $43 \%$ & $24 \%$ & $24 \%$ & 2.38 & $0.97(1$ to 4$)$ \\
\hline \multirow{2}{*}{$\begin{array}{l}\text { Expected outcome of periodontal } \\
\text { treatment }\end{array}$} & $0 \%$ & $14 \%$ & $62 \%$ & $24 \%$ & 1.90 & 0.63 (I to 3$)$ \\
\hline & $\begin{array}{l}\text { I = Strongly } \\
\text { Disagree }\end{array}$ & $2=$ Disagree & $3=$ Neutral & $4=$ Agree & $\begin{array}{c}5=\text { Strongly } \\
\text { Agree }\end{array}$ & Mean \\
\hline $\begin{array}{l}\text { Smile-related quality of } \\
\text { life of subjects } \\
\text { a. My teeth and gums cause discomfort. }\end{array}$ & $33 \%$ & $19 \%$ & $29 \%$ & $5 \%$ & $15 \%$ & 2.48 \\
\hline $\begin{array}{l}\text { b. My teeth and gums reduce my } \\
\text { general happiness with life. }\end{array}$ & $62 \%$ & $10 \%$ & $14 \%$ & $14 \%$ & $0 \%$ & 1.81 \\
\hline $\begin{array}{l}\text { c. My teeth and gums affect my life in } \\
\text { all of its aspects. }\end{array}$ & $71 \%$ & $5 \%$ & $5 \%$ & $14 \%$ & $5 \%$ & 1.76 \\
\hline $\begin{array}{l}\text { d. My teeth and gums keep me from } \\
\text { smiling. }\end{array}$ & $43 \%$ & $15 \%$ & $19 \%$ & $19 \%$ & $5 \%$ & 2.29 \\
\hline e. I like my smile. & $14 \%$ & $5 \%$ & $38 \%$ & $19 \%$ & $24 \%$ & 3.33 \\
\hline $\begin{array}{l}\text { f. I am happy with the looks of my } \\
\text { teeth and gums. }\end{array}$ & $10 \%$ & $10 \%$ & $33 \%$ & $38 \%$ & $10 \%$ & 3.29 \\
\hline
\end{tabular}

Percentages and descriptive statistics are based on the data collected from 21 patients. 
assessments of the periodontal health status of the subjects ranged from "poor" to "excellent." In addition, the graduate periodontal residents were asked to describe the outcome of the periodontal treatment. The residents anticipated that $61.9 \%$ of the subjects ultimately would have a good treatment outcome and that $23.8 \%$ would achieve an excellent outcome (Table 1). In addition, the frequencies of subject responses to the six quality-of-life statements also mirrored the variability that was found for the other two sets of indicators. Although a considerable percentage of subjects strongly disagreed or disagreed with these statements, some subjects agreed or even strongly agreed with these statements. In summary, although the sample size was small $(N=21)$, the respondents differed considerably and had a wide range of responses.

Table 2 provides an overview of the relationships between the four sets of indicators. There was a significant relationship between the providers' measurements of their subjects' periodontal health and their subjective assessments of their subjects' current periodontal health status and even the anticipated outcome of the periodontal treatment. The more teeth with probing depths $\geq 6 \mathrm{~mm}$ the subjects had, the more negatively their providers evaluated their periodontal health $(\mathrm{r}=0.595 ; P=0.004)$. The more teeth with mobility and the more missing teeth the subjects had, the worse the providers described their periodontal health $(\mathrm{r}=0.576$ and $P=0.006 ; \mathrm{r}=0.502$ and $P=0.020$ ) and the poorer the treatment outcome that was being predicted $(\mathrm{r}=0.680$ and $P<0.001 ; \mathrm{r}=$ 0.644 and $P=0.001$ ). The degree of gingival recession in the esthetic zone did not correlate significantly with the providers' subjective assessments of their subjects' periodontal health, indicating that providers might focus primarily on probing depth, teeth with mobility, and missing teeth when forming their global, subjective assessments of their subjects' periodontal health. In addition, the number of restored teeth did not correlate with the providers' subjective assessments of their subjects' current or future periodontal health status, further supporting the notion that the provider assessments are valid indicators of periodontal health and not merely assessments of oral health in general.

Table 2.

\section{Correlation Coefficients Among Periodontal Health, Provider Assessments, Quality-of-Life Scores, and Smiling Patterns}

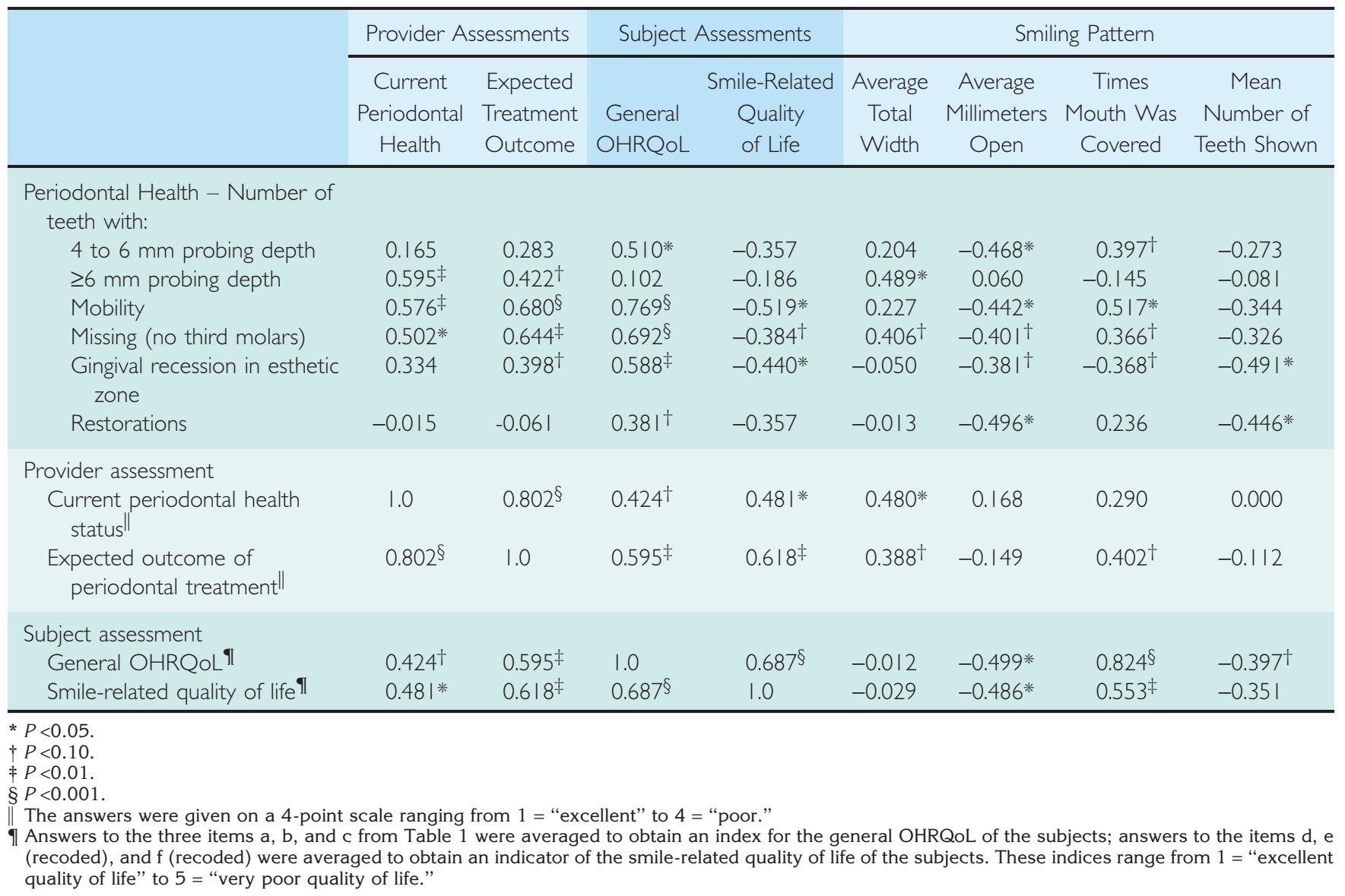


The second set of relationships considered was between the quality-of-life scores of the subjects and their clinically and subjectively assessed periodontal health indicators. As can be seen in Table 2, the subjects' ratings of their general OHRQoL and of their smile-related quality of life correlated significantly with their providers' subjective assessments of their current periodontal health $(\mathrm{r}=0.424$ and $P<0.10$; $\mathrm{r}=0.481$ and $P<0.05$, respectively) as well as with the provider assessments of the outcomes of the periodontal treatment $(\mathrm{r}=0.595$ and $P<0.01 ; \mathrm{r}=0.618$ and $P<0.01$, respectively). In addition, the general OHRQoL indicators correlated significantly with the number of teeth with probing depth 4 to $6 \mathrm{~mm} \mathrm{(r=}$ $0.510 ; P<0.05)$, the number of teeth with mobility $(\mathrm{r}=0.769 ; P<0.001)$, the number of missing teeth $(\mathrm{r}=0.692 ; P<0.001)$, and the number of teeth with gingival recession in the esthetic zone $(r=0.588$; $P<0.01)$. In addition, there was a tendency toward significance for the relationship between general OHRQoL and the number of restored teeth $(r=$ $0.381 ; P<0.10$ ), indicating that the subjects' OHRQoL assessments might take into considerations all indicators of oral health. The smile-related quality of life of the subjects correlated significantly with the number of teeth with mobility $(r=0.519 ; P<0.05)$ and, not surprisingly, the number of teeth with gingival recession in the esthetic zone $(r=0.440 ; P<0.05)$.

Finally, the relationships between the clinically and subjectively assessed periodontal health indicators, the quality-of-life assessments of the subjects, and the smiling patterns were considered. As can be seen in Table 2, the more teeth with 4 to $6 \mathrm{~mm}$ probing depth the subjects had, the less widely they opened their mouths $(r=-0.468 ; P<0.05)$ and the more frequently they tended to cover their mouths $(r=0.397$; $P<0.10)$. The more hypermobile teeth the subjects had, the less widely they opened their mouths when they smiled $(r=-0.442 ; P<0.05)$ and the more likely they were to cover their mouths with their hands $(r=$ $0.517 ; P=0.017)$. The more teeth with recession in the esthetic zone the subjects had, the fewer teeth they showed when they smiled $(\mathrm{r}=-0.491 ; P<$ $0.001)$. Finally, the more restored teeth the subjects had, the less open was their smile $(r=-0.496 ; P<$ $0.001)$. In summary, the clinically assessed oral health indicators of the subjects were related significantly to their smiling patterns.

The more the subjects reported that their general OHRQoL was impaired, the less open were their smiles $(r=-0.499 ; P<0.001)$, the more likely they were to cover their teeth $(r=0.824 ; P<0.001)$, and the fewer teeth they tended to show when they smiled $(r=$ $-0.397 ; P<0.10)$. In addition, the more the subjects reported that their smile-related quality of life was impaired, the less they opened their mouths when they smiled $(\mathrm{r}=-0.486 ; P<0.001)$ and the more often they covered up their mouths when they smiled $(r=0.553$; $P<0.01)$.

Finally, the relationship between the providers' subjective assessments of their subjects' periodontal health and the smile indicators showed that the poorer the providers evaluated their subjects' smiles to be, the wider their smiles were $(r=0.480 ; P<0.05)$. However, no significant relationships were found between this subjective health indicator and the other aspects of the subjects' smiling patterns.

\section{DISCUSSION}

Despite the fact that some degree of periodontal disease is seen in $>50 \%$ of the United States adult population, only three published studies ${ }^{12-14}$ have explored the relationship between periodontal disease and the quality of life of subjects. The findings of this study support the conclusions of these three earlier studies, i.e., that poor periodontal health affects the OHRQoL of subjects. The subjectively and clinically assessed health indicators correlated significantly with the OHRQoL and smile-related quality of life of the subjects, providing further support for the hypothesis that periodontal health affects the quality of life of subjects.

In addition, to the best of our knowledge, these findings also provide the first evidence that periodontal disease might affect the smiling patterns of subjects. The fact that there are significant correlations between the clinically assessed oral health indicators of subjects and the objectively determined smiling patterns is interesting: in particular, even an indicator of milder periodontal problems, e.g., the number of shallower pockets, correlated with smile indicators. For example, the negative correlation between the number of teeth with 4 to $6 \mathrm{~mm}$ probing depth and the average "millimeters open" showed, as predicted, that the more teeth with 4 to $6 \mathrm{~mm}$ probing depth the subjects had, the less wide/the fewer millimeters wide the subjects opened their mouths. In addition, the positive correlation between this oral health indicator and the number of times the subjects covered their mouths was also predicted: the more teeth with 4 to $6 \mathrm{~mm}$ probing depth the subjects had, the more frequently they covered their mouths. It is interesting that the correlations between the number of teeth with $>6 \mathrm{~mm}$ probing depth and these two smile indicators were not significant, whereas the correlation between this indicator of more severe periodontal issues correlated, as predicted, with the width of the mouths of the subjects in millimeters. This finding could be related to the possibility that subjects will smile by opening their mouth vertically, not horizontally, to cover problematic oral health issues. Future research should analyze these aspects of smiling patterns in more detail. 
Although Patel et al. ${ }^{15}$ showed that children's oral health status affected their smiling patterns, no published research has analyzed the effects of periodontal health on the smiles of adult subjects. Given that smiling has important effects on social interactions ${ }^{16}$ and the well-being and self-esteem of subjects, ${ }^{17}$ it seems worthwhile to consider the role of periodontal health on smiling behavior. Although dental care providers might easily concede that tooth loss or malocclusion can affect the smiling patterns of subjects, the fact that periodontal disease might affect smiling behavior might not be seen as obvious. Nevertheless, to the best of our knowledge, these data provide the first evidence that periodontal disease plays a role in the smile-related quality of life of subjects and even their actual smiling patterns.

Given these findings, it might be worthwhile to discuss the age- and gender-specific aspects of smiling patterns and how these changes may affect the present findings. In 1998, Zachrisson ${ }^{21}$ discussed the esthetic factors involved in anterior tooth display and the smile and pointed out that age-related changes and gender differences should be considered. A study of the vertical dimension of smiles by Vig and Brundo 22 showed that a gradual decrease in maxillary incisor exposure occurs over the years (from $<30$ years of age on), with increasing mandibular incisor display in subjects $>60$ years of age. However, these investigators argued that the amount of mandibular incisor display after age 60 was approximately equal to the amount of maxillary incisor display before age 30, which implies that the measures used in this study would not necessarily be sensitive to these age-related changes. However, given that the participants in this study ranged from 24 to 82 years of age, it seemed interesting to determine whether there was a relationship between age and clinically assessed oral health indicators, especially the average number of millimeters that the mouth was open and the number of teeth shown. The correlations between age and average millimeters open $(r=0.223)$ and the number of teeth shown $(r=$ 0.277 ) were not significant. Nevertheless, future research might consider measuring the vertical dimension of the smiles of subjects more specifically.

In addition, Zachrisson ${ }^{21}$ also discussed research on gender differences in smiling patterns. He reported that several studies ${ }^{22-24}$ found significant gender differences in anterior tooth display. Generally, females had significantly more maxillary and significantly less mandibular tooth exposure than males at all ages. More specifically, in an adult sample, Vig and Brundo 22 found almost twice as much maxillary anterior tooth display with the lips at rest in women $(3.4 \mathrm{~mm})$ than in men $(1.9 \mathrm{~mm})$. In addition, men displayed more of the mandibular incisors compared to women (1.2 $\mathrm{mm}$ versus $0.5 \mathrm{~mm}$ ). Again, it seemed worthwhile to explore whether the 17 women and four men participating in the present study systematically differed in these characteristics. Therefore, men's and women's average smiling indicators were compared to $t$ tests for independent samples. As in the earlier research, women opened their mouths significantly wider than men $(1.03 \mathrm{~mm}$ versus $0.026 \mathrm{~mm} ; P=$ $0.026)$ and showed more teeth on average when smiling (0.84 teeth versus 0.14 teeth; $P=0.01)$. Given these gender differences, the correlations between the four sets of indicators were computed for the female participants only. The pattern of gender-specific results was the same as for the combined sample. Given that data had been collected from only four male participants, a separate analysis of the male data was not conducted because of the small sample size. However, future research should explore gender differences.

In addition to exploring the effect of the age and gender of subjects, it also seems interesting to explore how provider and subject responses differ. For example, periodontal residents did not seem to consider the number of restored teeth nor the degree of gingival recession in the esthetic zone when assessing their subjects' oral health subjectively (Table 2 ). However, subjects seemed to consider these two indicators when describing their quality of life; for example, the degree of gingival recession in the esthetic zone correlated significantly with their general OHRQoL (Table 2). To improve subject-provider communication, it might be interesting to explore if there is consistency in the factors that providers and subjects use when subjectively determining a subject's periodontal health.

\section{CONCLUSIONS}

To the best of our knowledge, these findings provide the first empirical evidence that the periodontal health of subjects affects their OHRQoL in general, their smile-related quality of life, and their objectively determined smiling patterns. Although these findings were based on data from only 21 subjects because of the time-intensive nature of the assessments of the subjects' smiling patterns, it seems worthwhile to consider follow-up studies to explore these relationships in greater depth. Future research also might explore the effects of periodontal therapy on subjects' objective smiling patterns and subjective quality-oflife responses. Gaining a clearer understanding of how periodontal health/disease affects the lives of subjects follows the United States Surgeon General's recommendation to consider the ways in which oral disease shapes the quality of life of subjects. ${ }^{1}$

\section{ACKNOWLEDGMENTS}

The authors thank Dr. Laurie K. McCauley, chair, Department of Periodontics and Oral Medicine, School of 
Dentistry, University of Michigan, for her support of this research. We also thank the following research assistants and undergraduate students at the University of Michigan: Carli Cross, Kate Essad, and Gloria Lee for helping with the collection of the data and Sheri Boyd and Brittany Williams for rating the digitized videos of the smiling pattern. This research was supported by funding from the Department of Periodontics and Oral Medicine, School of Dentistry, University of Michigan, to Dr. Patel. The authors report no conflicts of interest related to this study.

\section{REFERENCES}

1. U.S. Department of Health and Human Services. Oral Health in America: A Report of the Surgeon General. Bethesda; MD: National Institute of Dental and Craniofacial Research, National Institutes of Health; 2000. NIH publication 00-4713.

2. Inglehart MR, Bagramian RA, eds. Oral Health and Quality of Life. Chicago: Quintessence; 2002.

3. Inglehart MR, Bagramian RA. Oral health-related quality of life - Introduction and overview. In: Inglehart MR, Bagramian RA, eds. Oral Health and Quality of Life. Chicago: Quintessence; 2002:1-6.

4. Ellis JS, Pelekis ND, Thomason JM. Conventional rehabilitation of edentulous patients: The impact on oral health-related quality of life and patient satisfaction. J Prosthodont 2007; 16:37-42.

5. Tsakos G, Steele JG, Marcenes W, Walls AW, Sheiham A. Clinical correlates of oral health-related quality of life: Evidence from a national sample of British older people. Eur J Oral Sci 2006;114:391-395.

6. Koshino $H$, Hirai $T$, Ishijima $T$, Tsukagoshi $H$, Ishigami T, Tanaka Y. Quality of life and masticatory function in denture wearers. J Oral Rehabil 2006;33: 323-329.

7. Zhang M, McGrath C, Hagg U. Patients' expectations and experiences of fixed orthodontic appliance therapy. Impact on quality of life. Angle Orthod 2007;77: 318-322

8. Zhang M, McGrath C, Hagg U. The impact of malocclusion and its treatment on quality of life: A literature review. Int $J$ Paediatr Dent 2006;16:381-387.

9. Low W, Tan S, Schwartz S. The effect of severe caries on the quality of life in young children. Pediatr Dent 1999;21:325-326.

10. Filstrup SL, Briskie D, da Fonseca M, Lawrence L, Wandera A, Inglehart MR. Early childhood caries and quality of life - Child and parent perspectives. Pediatr Dent 2003;25:431-440.

11. Sheiham A. Dental caries affects body weight, growth and quality of life in pre-school children. Br Dent $J$ 2006;201:625-626.

12. Needleman I, McGrath C, Floyd P, Biddle A. Impact of oral health on the life quality of periodontal patients. $J$ Clin Periodontol 2004;31:454-457.

13. Ng SK, Leung WK. Oral health-related quality of life and periodontal status. Community Dent Oral Epidemiol 2006;34:114-122.

14. Cunha-Cruz J, Hujoel PP, Kressin NR. Oral healthrelated quality of life of periodontal patients. J Periodontal Res 2007;42:169-176.

15. Patel RR, Tootla R, Inglehart MR. Children's smiling patterns - A function of oral health? Community Dent Oral Epidemiol 2007;35:44-52.

16. Reis HT, Wilson IM, Monestere C. What is smiling is beautiful and good. Eur J Soc Psychol 1990;20:259-267.

17. Tamir M, Robinson MD, Clore GL, Martin LL, Whitaker DJ. Are we puppets on a string? The contextual meaning of unconscious expressive cues. Pers Soc Psychol Bull 2004;30:237-249.

18. Källestål C, Dahlgren L, Stenlund H. Oral health behaviour and self-esteem in Swedish children. Soc Sci Med 2000;51:1841-1849.

19. Henson B, Inglehart MR, Eisbruch A, Ship J. Preserved salivary output and xerostomia-related quality of life in head and neck cancer patients receiving parotidsparing radiotherapy. Oral Oncol 2001;37:84-93.

20. Ekman P, Rosenberg EL, eds. What the Face Reveals. Basic and Applied Studies of Spontaneous Expression Using the Facial Action Coding System (FACS), 2nd ed. Oxford, U.K.: Oxford University Press; 2005.

21. Zachrisson BU. Esthetic factors involved in anterior tooth display and the smile: Vertical dimension. J Clin Orthod 1998;32:432-445.

22. Vig RG, Brundo GC. The kinetics of anterior tooth display. J Prosthet Dent 1978;39:502-504.

23. Peck S, Peck L, Kataja M. The gingival smile line. Angle Orthod 1992;62:91-100.

24. Peck S, Peck L, Kataja M. Some vertical lineaments of lip position. Am J Orthod Dentofacial Orthop 1992; 101:519-524.

Correspondence: Dr. Marita R. Inglehart, Department of Periodontics and Oral Medicine, School of Dentistry, University of Michigan, 1011 N. University, Ann Arbor, MI 48109-1078. Fax: 734/763-5503; e-mail: mri@umich.edu.

Submitted June 17, 2007; accepted for publication August 3, 2007. 